\title{
DEM's elevation comparison by surface buffering
}

\author{
Ariza-López, F.J. ${ }^{\mathrm{a},}$ *, Rodríguez-Avi, J. ${ }^{\mathrm{a}}$, Reinoso-Gordo, J.F. b \\ a University of Jaén, fjariza@ujaen.es; jravi@ujaen.es \\ ${ }^{b}$ University of Granada, jreinoso@ugr.es \\ * Corresponding author
}

Keywords: DEM, data quality, buffering

\begin{abstract}
:
Traditionally, the altimetric accuracy assessment of digital elevation models (DEM) has been carried out using methods based on sampling of homologous points in a reference model (S1) and in the model to be assessed (S2). These methods have two drawbacks: a) the homologous points are scarce compared to the total surface of a DEM and therefore they leave a large ground area not assessed, 2) the DEMs assessment is carried out comparing points, when it seems more suitable to assess by a surface comparison. Both drawbacks can be overcome by surface buffering methods. This work presents the use of surface buffering methods in order to compare gridded DEM. The study has been carried out on synthetic data.

In the case of line-based data (e.g., roads, coastlines, etc.), in which it is not always possible to find well-defined or homologous points between two representations of the same reality, specific evaluation methods have been proposed based on buffering techniques, such as the single buffer method (SBM) by Goodchild and Hunter (1997) and the double buffer method (DBM) by Tveite (1999).

The objective of this work is to apply the two previously mentioned buffer-based methods to DEM surfaces with the aim of comparing the altimetric behavior of two DEM data sets. With this new perspective, the inconvenience of not having well-defined points in the case of grid-type DEMs is avoided and, furthermore, it can be applied in the natural context of the reality being analyzed, which is that of a surface, actually a $2.5 \mathrm{D}$ surface. Methods based on buffers can be used to compare two DEM data sets, or a product and a reference (quality control).

We use two synthetic data sets because in order to have a controlled design of our experiment. The synthetic data sets are shown in Figure 1. It is about two surfaces: S1 and S2. S1 is sinusoidal in nature and consists of a lower frequency carrier on which another higher frequency sinusoidal pattern mounts. S2 is a plane of constant slope. S1 can be considered as a better approximation to reality since it has greater detail, while S2 can be considered as a generalization. Thus, in the case of a positional quality control, S1 would act as a reference and S2 as a product. The configuration of S1 and S2 allows you to observe what happens to the undulations and the bias that exists between the two surfaces.
\end{abstract}

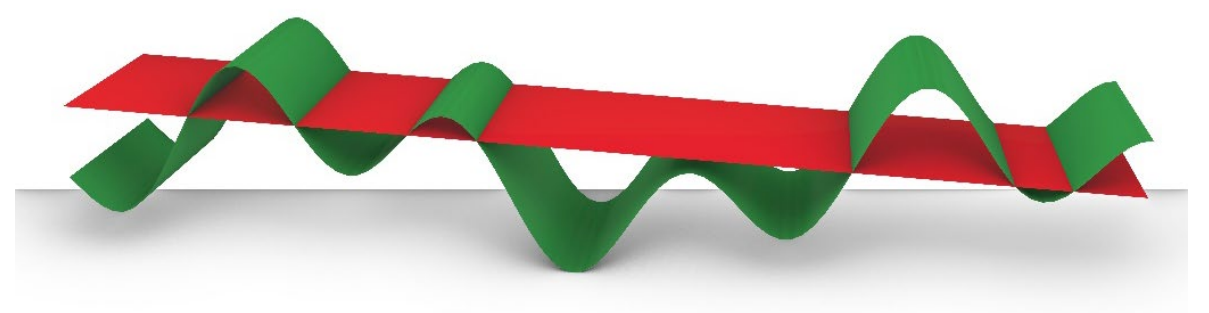

Figure 1. The two DEM synthetic data sets for the experiment: S1 in green (reference) and S2 in red (product).

Our approach is based on a direct translation of the SBM and DBM to gridded DEMs by means of the use of voxels. By this way interesting results have been achieved. In relation to the SBM, Figure 2 shows the evolution of the percentage of the S2 DEM's surface included when S1 is buffered with a semi-width $(w)$. This curve allows you to recognize the presence/absence of outliers. When comparing two DEMs, outliers must meet two conditions, be large discrepancies and have a small frequency of occurrence. So that, an outlier would be an excessively large discrepancy between S1 and S2, this means large values of $w$, and for the frequency to be small, an asymptotic behavior is required. This presence would generate the need for a very large value of $w$ to achieve its inclusion and an asymptotic flattening of the curve. As can be seen in Figure 2, this is not the case, so there is no presence of outliers. Also, the curve allows us to recognize the presence/absence of vertical bias. The vertical bias in this case means that one DEM has altimetric predominance over the other. In our case, there is bias: S2 > S1, and is evidenced by $20 \%$ of surface imbalance. This value is deduced from the following operation: $\mathrm{F}(\mathrm{w}=6 \mathrm{~m})-\mathrm{F}(\mathrm{w}=4 \mathrm{~m}) . \mathrm{w}=6$ is the mean value of the border range and $\mathrm{w}=4 \mathrm{~m}$ is the border width value for which we have half the accumulated frequency. 


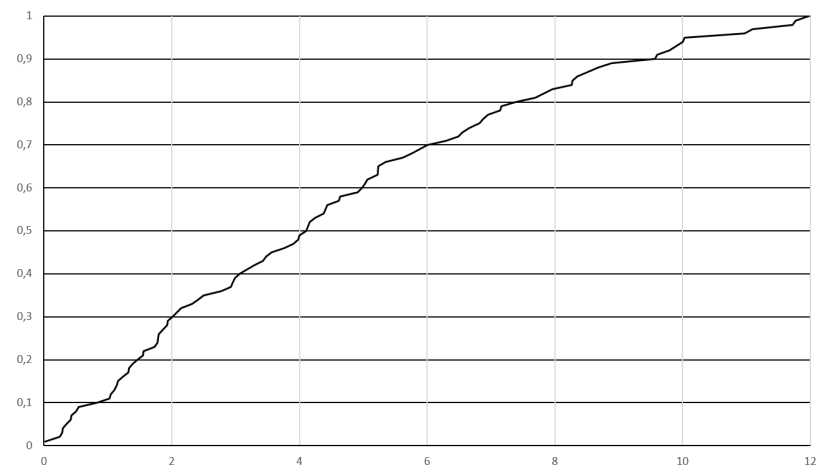

Figure 2. Result of applying the SBM. Vertical axis: degree of S1-DEM's surface inclusion for each buffer semi-width (w) (horizontal axis).

The results for the DBM are more complete and complex. Figure 3a shows the evolution of the S1-DEM's surface percentage of inclusion for different semi-widths $[1,3,5,10,15,20]$. The horizontal axis corresponds to the percentage of common intersection between voxels of S1 and S2 (degree of voxel-overlapping), and the vertical axis to the percentage of area of the DEM zone included by each $w$ and voxel-overlapping value. The evolution of these curves can also be represented spatially, which helps to better understand the behavior of the discrepancies between S2 and S1 (e.g., the presence of spatial groupings or areas with different behaviors in the degree of voxel-overlapping). Figure $3 \mathrm{~b}$ shows several curves, the solid one is equivalent to that of figure 2, but for the DBM. Dashed lines represent the evolution of the S1-DEM's surface percentage above (- -) and below (- -) S2. As can be seen, Figure 3.b clearly shows the existence of a bias between S2 and S1 and that it is in the order of $20 \%$ of the frequency (see starting values of the curves above and below). These types of curves would also show the presence of outliers, if they existed.
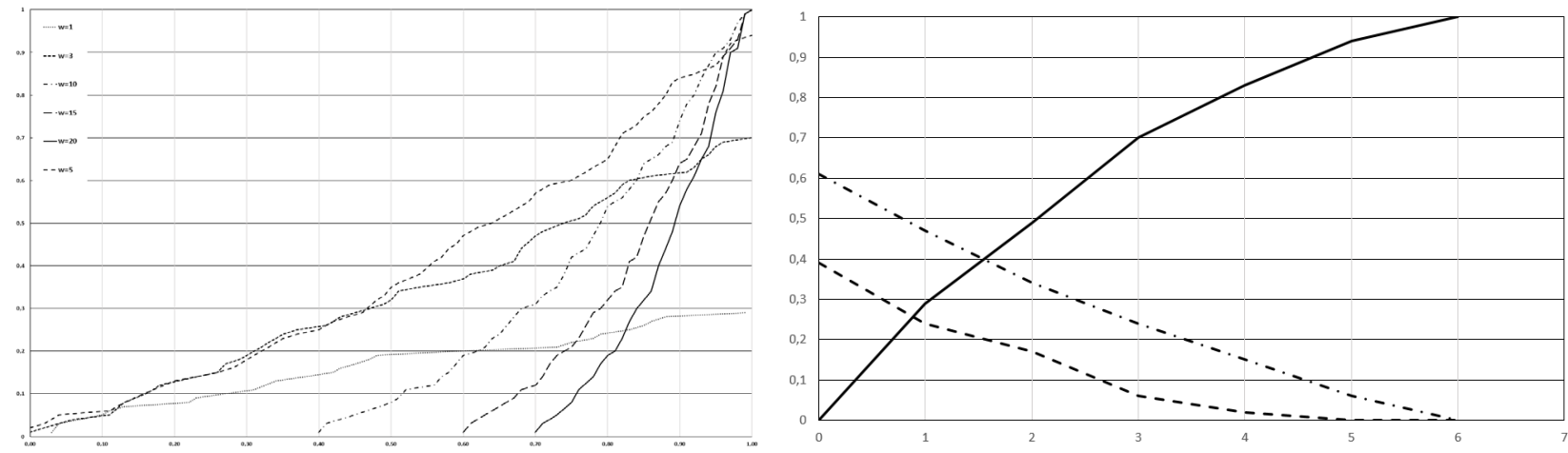

Figure 3. Result of applying the DBM. 3.a (left) degree of S1-DEM's surface inclusion for each buffer semi-width (w). 3.b (right) degree of S1-DEM's surface inclusion above (- - -) and below (- -) of S2, and total (-).

The application of the SBM and DBM to the case of gridded MDEs is quite straightforward, although it requires some adaptation but, in any case, they are solved with simple map algebra operations. The results of the SBM allow a direct relationship with methods of positional accuracy quality control already developed, and that are based on control by tolerances applying multinomials. In addition, the surface inclusion curves allow conclusions to be drawn about the presence of outliers and biases. The results of the DBM are richer and more complex than those of the SBM since it is played with the degree of intersection of the voxels of the two surfaces, which allows a probabilistic interpretation of the inclusion percentage of the surfaces that, in addition, can help visual interpretation. This method also makes it possible to analyze the presence of biases. The results of both methods are complementary to those that already exist, since each one presents a different perspective.

This work is a first approximation and therefore is based on synthetic data in limited quantity and type. Along with the extension of these tests, the application to the case of real data is also pending. We consider that these two methods may be of great interest for comparing the altimetry of several DEMs of the same area, given that nowadays it is increasingly common to have DEMs coming from different sources for the same area. These comparisons may be of environmental interest (e.g., erosion analysis, slope landslide analysis, etc.).

Goodchild M.F., Hunter G. (1997). A simple positional accuracy measure for linear features. International Journal of Geographical Information Science, 11(3), pp. 206-299, DOI 10.1080/136588197242419

Tveite, H. (1999) An accuracy assessment method for geographical line data sets based on buffering, International Journal of Geographical Information Science, 13(1), pp. 27-47, DOI: 10.1080/136588199241445 